\title{
A geometric definition of the Aubry-Mather set
}

\author{
Patrick Bernard* and Joana Oliveira dos Santos
}

February 2010

Patrick Bernard, Université Paris-Dauphine, CEREMADE, UMR CNRS 7534

Pl. du Maréchal de Lattre de Tassigny

75775 Paris Cedex 16, France

patrick.bernard@ceremade.dauphine.fr
Joana O. Santos, Université Paris-Dauphine, CEREMADE, UMR CNRS 7534

Pl. du Maréchal de Lattre de Tassigny

75775 Paris Cedex 16, France

santos@ceremade.dauphine.fr

Abstract. We study the Aubry set which appears in Mather theory of convex Hamiltonian systems from the point of view of symplectic geometry.

Résumé. On étudie l'ensemble d'Aubry qui apparait dans la théorie de Mather des systèmes Hamiltoniens convexes du point de vue de la géométrie symplectique.

Resumo Estudamos o conjunto de Aubry, que aparece na teoria de Mather em sistemas Hamiltonianos convexos, do ponto de vista da geometria simplética.

MSC: 37J50, 37J05, 53D12, 57R17.

${ }^{*}$ membre de l'IUF 


\section{Introduction}

We consider a $C^{2}$ Hamiltonian $H: T^{*} M \longrightarrow \mathbb{R}$ on the cotangent bundle of a compact manifold $M$ (of dimension $n$ ) without boundary. Most of our results will be relevant only in the case where $H$ is optical, which means that it is convex with positive definite Hessian and superlinear in each fiber. It is well known that the dynamics of optical Hamiltonians can be studied with the use of the Lagrangian action functional. Using this approach, Mather defined an important compact invariant set, that is now called the Aubry set, and which has the distinguished property of being contained in a Lipschitz Lagrangian graph, see [13]. This set, and the corresponding notion of critical value, has been studied in a number of papers, In the present paper, we study it from a geometric point of view and give new proofs of some of its most important properties: dynamical invariance, symplectic invariance, and the graph property (which is tautological for the definition we use).

Let $\mathcal{G}$ be the set of Lipschitz exact Lagrangian graphs in $T^{*} M$. An element of $\mathcal{G}$ is a subset $\Gamma \subset T^{*} M$ of the form

$$
\Gamma:=\{(x, d u(x)), x \in M\}
$$

where $u$ is a $C^{1,1}$ function (a $C^{1}$ function with Lipschitz differential). To a given Hamiltonian $H$, we associate its critical value

$$
\alpha(H):=\inf _{\Gamma \in \mathcal{G}} \max _{(x, p) \in \Gamma} H(x, p) .
$$

This definition was introduced in [8] where it is also proved that the value $\alpha(H)$ would not be changed if the regularity of the graphs considered in the infimum was increased. The Lipschitz regularity has the advantage of being the highest one for which the infimum is a minimum, see [4]. We define the Aubry set

$$
\tilde{\mathcal{A}}(H):=\bigcap_{\Gamma \in \mathcal{G}, \Gamma \subset\{H \leqslant \alpha(H)\}} \Gamma=\bigcap_{\Gamma \in \mathcal{G}, \Gamma \subset\{H \leqslant \alpha(H)\}}(\Gamma \cap\{H=\alpha(H)\}) .
$$

Let us prove the second equality. We consider an element $\Gamma=\{(x, d u(x)), x \in M\}$ of $\mathcal{G}$ contained in $\{H \leqslant \alpha(H)\}$, and a point $x_{0} \in M$ such that $H\left(x_{0}, d u\left(x_{0}\right)\right)<\alpha(H)$. We want to prove that there exists an element $\Gamma_{1}$ of $\mathcal{G}$ contained in $\{H \leqslant \alpha(H)\}$ and not containing the point $\left(x_{0}, d u\left(x_{0}\right)\right)$. In order to do so, we consider a $C^{1,1}$ function $v$ which is equal to $u$ outside of a small neighborhood of $x_{0}$, which is $C^{1}$-close to $u$, and such that $d v\left(x_{0}\right)$ is different from $d u\left(x_{0}\right)$. We can chose such a function $v$ in such a way that the inequality $H(x, d v(x)) \leqslant \alpha(H)$ holds everywhere. Then the Lagrangian graph $\Gamma_{1}:=\{(x, d v(x)), x \in M\}$, satisfies all the required properties.

The first step of the theory, which is also the hardest one, consists in proving that the Aubry set $\tilde{\mathcal{A}}(H)$ of an optical Hamiltonian is not empty. It is already a non-trivial fact that the set of elements $\Gamma \in \mathcal{G}$ which are contained in $\{H \leqslant \alpha(H)\}$ is not empty, or in other words that the infimum in the definition of $\alpha(H)$ is a minimum. The following result was proved in [4] extending an earlier result of [10] (see [1] for a more geometric presentation of the proof) :

Theorem 1. If $H$ is optical, then there exists an element $\Gamma \in \mathcal{G}$ such that $H_{\mid \Gamma} \leqslant \alpha(H)$ and such that $\tilde{\mathcal{A}}(H)=\Gamma \cap\{H=\alpha(H)\}$. As a consequence, the set $\tilde{\mathcal{A}}(H)$ is not empty.

It is also well-known that the set $\tilde{\mathcal{A}}(H)$ is invariant under the Hamiltonian flow. Moreover, it is a symplectic invariant, as is proved in [5]( some relations between symplectic geometry and Mather theory are also studied in $[14,17])$ :

Theorem 2. Let $H$ be an optical Hamiltonian, and let $\varphi$ be an exact symplectic diffeomorphism of $T^{*} M$ such that $H \circ \varphi$ is also optical. Then

$$
\alpha(H \circ \varphi)=\alpha(H) \quad \text { and } \quad \varphi(\tilde{\mathcal{A}}(H \circ \varphi))=\tilde{\mathcal{A}}(H) .
$$


This result is not a trivial consequence of the definition. The proof given in [5] uses another approach to the Aubry set, closer to the original definition of Mather. Our goal in the present paper is to try to understand this result directly on the presented definition. We shall be able to reach this goal only when $\varphi$ is a Hamiltonian diffeomorphism. It is an open and intriguing question to prove the full statement by the geometric methods we are going to discuss below.

It is worth observing that Theorem 2 directly implies the dynamical invariance of $\tilde{\mathcal{A}}(H)$. It is enough to apply it when $\varphi$ is the Hamiltonian flow (which is a Hamiltonian diffeomorphism, so that our "restricted" version is enough).

In the present paper, we will slightly modify the definition of the Aubry set above and define a new set in such a way that symplectic invariance under Hamiltonian diffeomorphisms (and thus dynamical invariance) is a tautological consequence of the definition. Then, we will prove that this new Aubry set coincides with the classical one defined above in the case of optical Hamiltonians. This class unfortunately remains at this point the only one for which we can prove that the Aubry set is not empty. Some of the methods and results are similar to those introduced by Paternain, Polterovich and Siburg in [14], this paper is the starting point of the present work.

\section{Generalized Aubry sets}

Let $\mathcal{L}$ be a set of compact subsets of $T^{*} M(\mathcal{L}=\mathcal{G}$ for example $)$. We define the critical value

$$
\alpha_{\mathcal{L}}(H):=\inf _{L \in \mathcal{L}} \max _{(x, p) \in L} H(x, p)
$$

and the set

$$
\tilde{\mathcal{A}}_{\mathcal{L}}(H):=\bigcap_{L \in \mathcal{L}, L \subset\left\{H \leqslant \alpha_{\mathcal{L}}(H)\right\}} L
$$

It may happen that the set of elements $L \in \mathcal{L}$ contained in the sublevel $\left\{H \leqslant \alpha_{\mathcal{L}}(H)\right\}$ is empty, in which case we define $\tilde{\mathcal{A}}_{\mathcal{L}}(H)$ as the empty set. Of course, the theory will be interesting only in the situations where we are able to prove that $\tilde{\mathcal{A}}_{\mathcal{L}}(H)$ is not empty. In the case $\mathcal{L}=\mathcal{G}$, we have the property of local symplectic invariance:

For each $L \in \mathcal{L}$ and each Hamiltonian isotopy $\varphi^{t}$, there exists $\epsilon>0$ such that $\varphi^{t}(L) \in \mathcal{L}$ when $|t| \leqslant \epsilon$.

It is possible to deduce from this property that the Aubry set $\tilde{\mathcal{A}}(H)$ of an optical Hamiltonian is invariant under the Hamiltonian flow. In order to do so we follow [?] (another proof following the nice idea of [14], section 1.3.1 would also be possible). We consider the Hamiltonian isotopy $\varphi^{t}$ generated by $H$. Recall that $H \circ \varphi^{t}=H$. Let $\Gamma \in \mathcal{G}$ be such that $H_{\mid \Gamma} \leqslant \alpha(H)$ and $\tilde{\mathcal{A}}(H)=\Gamma \cap\{H=\alpha(H)\}$. Note that the existence of such a graph $\Gamma$ is given by Theorem 1 provided that $H$ is optical. Then, by local symplectic invariance, for $t$ small enough, we have $\varphi^{-t}(\Gamma) \in \mathcal{G}$ hence $\tilde{\mathcal{A}}(H) \subset \varphi^{-t}(\Gamma)$ and then

$$
\varphi^{t}(\tilde{\mathcal{A}}(H)) \subset \Gamma \cap\{H=\alpha(c)\}=\tilde{\mathcal{A}}(H) .
$$

As a consequence, the equality $\varphi^{t}(\tilde{\mathcal{A}}(H))=\tilde{\mathcal{A}}(H)$ holds when $t$ is small enough, but then it holds for all $t$.

We will be interested in sets $\mathcal{L}$ satisfying the stronger property of Hamiltonian invariance (resp. exact invariance):

For each $L \in \mathcal{L}$ and each Hamiltonian (resp. exact symplectic) diffeomorphism $\varphi$, we have $\varphi(L) \in \mathcal{L}$.

In the case $\mathcal{L}=\mathcal{G}$, Hamiltonian invariance does not hold, which prevents from using the following easy result to prove Theorem 2: 
Proposition 1. If $\mathcal{L}$ satisfies Hamiltonian (resp. exact) invariance, then, for each Hamiltonian (resp. exact symplectic) diffeomorphism $\varphi$, we have

$$
\alpha_{\mathcal{L}}(H \circ \varphi)=\alpha_{\mathcal{L}}(H) \quad \text { and } \quad \varphi\left(\tilde{\mathcal{A}}_{\mathcal{L}}(H \circ \varphi)\right)=\tilde{\mathcal{A}}_{\mathcal{L}}(H)
$$

As a consequence, the set $\tilde{\mathcal{A}}_{\mathcal{L}}(H)$ is invariant under the Hamiltonian flow.

Proof. Let $\varphi$ be a Hamiltonian (resp. exact symplectic) diffeomorphism. For each $L \in \mathcal{L}$ such that $H \circ \varphi_{\mid L} \leqslant \alpha_{\mathcal{L}}(H \circ \varphi)$, we have $\varphi(L) \in \mathcal{L}$ and $H_{\mid \varphi(L)} \leqslant \alpha_{\mathcal{L}}(H \circ \varphi)$. We conclude that the sublevel $\left\{H \leqslant \alpha_{\mathcal{L}}(H \circ \varphi)\right\}$ contains an element of $\mathcal{L}$, and therefore $\alpha_{\mathcal{L}}(H) \leqslant \alpha_{\mathcal{L}}(H \circ \varphi)$. We can apply this inequality with $H \circ \varphi$ instead of $H$ and $\varphi^{-1}$ instead of $\varphi$, and get that $\alpha_{\mathcal{L}}(H \circ \varphi) \leqslant \alpha_{\mathcal{L}}(H)$, hence $\alpha_{\mathcal{L}}(H)=\alpha_{\mathcal{L}}(H \circ \varphi)$. Then, we conclude that $\tilde{\mathcal{A}}_{\mathcal{L}}(H) \subset \varphi(L)$ for each $L \in \mathcal{L}$, and consequently that

$$
\tilde{\mathcal{A}}_{\mathcal{L}}(H) \subset \bigcap_{L \in \mathcal{L}, L \subset\left\{H \circ \varphi \leqslant \alpha_{\mathcal{L}}(H \circ \varphi)\right\}} \varphi(L)=\varphi\left(\bigcap_{L \in \mathcal{L}, L \subset\left\{H \circ \varphi \leqslant \alpha_{\mathcal{L}}(H \circ \varphi)\right\}} L\right)=\varphi\left(\tilde{\mathcal{A}}_{\mathcal{L}}(H \circ \varphi)\right) .
$$

Applying this result with $H \circ \varphi$ instead of $H$ and $\varphi^{-1}$ instead of $\varphi$, we get the reversed inclusion

$$
\tilde{\mathcal{A}}_{\mathcal{L}}(H \circ \varphi) \subset \varphi^{-1}\left(\tilde{\mathcal{A}}_{\mathcal{L}}(H)\right)
$$

and thus the equality. The dynamical invariance follows directly by applying this to the Hamiltonian flow.

\section{A symplectically invariant Aubry set}

In this section, we specify the abstract construction of Section 2 in the case where

$$
\mathcal{L}=\{\varphi(\Gamma), \Gamma \in \mathcal{G}, \varphi \in \mathcal{H}\}
$$

where $\mathcal{H}$ is the set of all Hamiltonian diffeomorphisms. By construction, this set $\mathcal{L}$ satisfies Hamiltonian invariance, and therefore the corresponding set $\tilde{\mathcal{A}}_{\mathcal{L}}(H)$ is invariant under Hamiltonian diffeomorphisms, and dynamically invariant. Our main statement is the following:

Theorem 3. If $H$ is an optical Hamiltonian, then for the set $\mathcal{L}$ defined in $\left({ }^{*}\right)$, we have $\alpha_{\mathcal{L}}(H)=$ $\alpha(H)$ and $\tilde{\mathcal{A}}_{\mathcal{L}}(H)=\tilde{\mathcal{A}}(H)$.

This theorem and Proposition 1 obviously imply Theorem 2 (restricted to Hamiltonian diffeomorphisms). Before proving Theorem 3, let us make some comments on regularity. We define by $\mathcal{G}^{\infty}$ and $\mathcal{L}^{\infty}$ the set of Lagrangian manifolds in $\mathcal{G}$ and $\mathcal{L}$ which are smooth. It was proved in [8] that

$$
\alpha_{\mathcal{G}}(H)=\alpha_{\mathcal{G}}(H)
$$

for all $C^{2}$ optical Hamiltonians $H$, and similarly, we have

$$
\alpha_{\mathcal{L}}(H)=\alpha_{\mathcal{L}^{\infty}}(H) .
$$

However, even in the case of optical Hamiltonians, the sets $\tilde{\mathcal{A}}_{\mathcal{G}}(H)$ and $\tilde{\mathcal{A}}_{\mathcal{L}^{\infty}}(H)$ are often empty because there is no element of $\mathcal{L}^{\infty}$ contained in $\left\{H \leqslant \alpha_{\mathcal{L}^{\infty}}(H)\right\}$, some examples are given in [4].

The proof of Theorem 3 is based on a property of the elements of $\mathcal{L}$ that we now describe. 


\subsection{Generalized graph selector}

Let us first recall the standard graph selector principle (see [7, 16]). There is a nice presentation in [14], where the idea of using the graph selector for the circle of problems we are considering is introduced. See also [2] for a very interesting related work.

Theorem 4. Let $L \subset T^{*} M$ be an exact Lagrangian manifold isotopic to the zero section, then $L$ admits a graph selector, that is a Lipschitz function $u: M \longrightarrow \mathbb{R}$ which is smooth on a dense open set $U \subset M$ of full measure, and such that $(x, d u(x)) \in L$ for each $x \in U$.

It is not clear whether the elements of $\mathcal{L}$ as defined in $\left(^{*}\right)$ also admit a graph selector. However, they admit a graph selector in a generalized sense. For each compact subset $K \subset T^{*} M$, we denote by $\hat{K}$ the fiberwise convexification of $K$. In other words, for each $x \in M$, the set $\hat{K} \cap T_{x}^{*} M$ is the convex hull of the set $K \cap T_{x}^{*} M$.

Lemma 1. For each compact set $K \subset T^{*} M$, the fiberwise convexification $\hat{K}$ of $K$ is compact.

Proof. Let $y_{k}=\left(x_{k}, p_{k}\right)$ be a sequence of points of $\hat{K}$. By the Theorem of Carathéodory (see [15], Theorem 17.1), for each $k$, there exist $n+1$ points $p_{k}^{i}, 0 \leqslant i \leqslant n$ in $K \cap T_{x_{k}}^{*} M$ and $n+1$ coefficients $t_{k}^{i}$ in $[0,1]$ such that $\sum_{i=0}^{n} t_{k}^{i}=1$ and such that $p_{k}=\sum_{i=0}^{n} t_{k}^{i} p_{k}^{i}$. Recall that $n$ is the dimension of $M$. By taking a subsequence, we can assume that each of the sequences $t_{k}^{i}$ converges to a limit $t^{i}$ in $[0,1]$, and that each of the sequences $\left(x_{k}, p_{k}^{i}\right)$ converges to $\left(x, p^{i}\right) \in K$, where $x$ is some point of $M$. Then, the sequence $y_{k}$ is converging to $\left(x, \sum_{i} t^{i} p^{i}\right)$, which belongs to $\hat{K}$.

Our generalization of the graph selector is the following:

Proposition 2. For each $L \in \mathcal{L}$, there exists a Lipschitz function $u$ on $M$ such that $(x, d u(x)) \in$ $\hat{L}$ for almost every $x$. We call this function u a generalized graph selector.

Proof. We have $L=\varphi(\Gamma)$, where $\varphi$ is a Hamiltonian diffeomorphism, and $\Gamma$ a graph of the form $\{(x, d v(x)), x \in M\}$, for some $C^{1,1}$ function $v$. Let $v_{k}$ be a sequence of smooth functions which converge to $v$ in $C^{1}$. The exact Lagrangian manifolds $L_{k}=\varphi\left(\left\{\left(x, d v_{k}(x)\right), x \in M\right\}\right)$ each admit a graph selector $u_{k}$. Since the manifolds $L_{k}$ are all contained in the same bounded subset of $T^{*} M$, and since we have $\left(x, d u_{k}(x)\right) \in L_{k}$ almost everywhere, we conclude that the sequence $u_{k}$ is equi-Lipschitz. By adding appropriate constants (which does not alter the property of being a graph selector), we can assume that the functions $u_{k}$ are equi-bounded, so that the sequence $u_{k}$ is bounded in the Sobolev space $W^{1, \infty}$. Therefore, we can assume by taking a subsequence that the functions $u_{k}$ converge weakly in $W^{1, \infty}$ to a limit $u$. We have to prove that $(x, d u(x)) \in \hat{L}$ for almost every $x$.

Let $L_{i}, i \in \mathbb{N}$ be a decreasing sequence of compact neighborhoods of $L$ such that $L=\cap_{i} L_{i}$. Let $\hat{L}_{i}$ be the fiberwise convexification of $L_{i}$. In the space $W^{1, \infty}(M)$ of Lipschitz functions on $M$, we consider the subset $F_{i}$ made of those functions $w$ such that $(x, d w(x)) \in \hat{L}_{i}$ for almost every $x$. The set $F_{i}$ is strongly closed and convex, thus weakly closed. Since $u_{k} \in F_{i}$ when $k$ is large enough, we conclude that $u \in F_{i}$, and this holds for each $i$, hence $u \in \cap_{i} F_{i}$. This means that, for each $i$, the inclusion $(x, d u(x)) \in \hat{L}_{i}$ holds outside of a set of measure zero, and then that the inclusion $(x, d u(x)) \in \cap_{i} \hat{L}_{i}$ holds almost everywhere. In order to finish the proof, it is enough to verify that $\cap_{i} \hat{L}_{i} \subset \hat{L}$. We take a point $(x, p) \in \cap_{i} \hat{L}_{i}$. By Carathéodory Theorem, for each $i$, there exist $n+1$ points $\left(x, p_{i}^{j}\right) \in L_{i}, 0 \leqslant j \leqslant n$ such that $p=\sum_{j=0}^{n} t_{i}^{j} p_{i}^{j}$, where $t_{i}^{j} \in[0,1]$ satisfy $\sum_{j=0}^{n} t_{i}^{j}=1$. By taking a subsequence in $i$, we consider that the sequences $t_{i}^{j}$ and $p_{i}^{j}$ converge as $i \longrightarrow \infty$. The limit $\left(x, p^{j}\right)$ of the sequence $\left(x, p_{i}^{j}\right)$ belongs to $L_{i}$ for each $i$ and therefore to $L$. Since $p=\sum_{j=0}^{n} t^{j} p^{j}$, we conclude that $(x, p) \in \hat{L}$. This proves that $\cap_{i} \hat{L}_{i} \subset \hat{L}$.

An important consequence is the following: 
Proposition 3. Let $H$ be an optical Hamiltonian. If the sublevel $\{H \leqslant c\}$ contains an element of $\mathcal{L}$, then it contains an element of $\mathcal{G}$.

Proof. If $L$ is contained in $\{H \leqslant c\}$, then so is $\hat{L}$. Taking a generalized graph selector $u$, we observe that $u$ is a Lipschitz solution of the Hamilton-Jacobi inequality

$$
H(x, d u(x)) \leqslant c
$$

The main result of [4] states that there exists a $C^{1,1}$ subsolution provided there exists a Lipschitz one. Applying this result, we find a $C^{1,1}$ subsolution $v$, the corresponding graph

$$
\Gamma=\{(x, d v(x)), x \in M\} \in \mathcal{G}
$$

is then contained in the sublevel $\{H \leqslant c\}$.

\subsection{Coincidence}

We now prove Theorem 3. Since $\mathcal{G} \subset \mathcal{L}$, we have $\alpha_{\mathcal{L}}(H) \leqslant \alpha(H)$. Conversely, if the inequality $\alpha(H)>\alpha_{\mathcal{L}}(H)$ was true, then picking some $c$ in ] $\alpha_{\mathcal{L}}(H), \alpha(H)$ [, the sublevel $\{H \leqslant c\}$ would contain an element of $\mathcal{L}$, and therefore, by Proposition 3, an element of $\mathcal{G}$. This is in contradiction with the definition of $\alpha(H)$. So we have $\alpha(H)=\alpha_{\mathcal{L}}(H)$.

As a consequence, recalling that $\mathcal{G} \subset \mathcal{L}$, we must have $\tilde{\mathcal{A}}_{\mathcal{L}}(H) \subset \tilde{\mathcal{A}}(H)$. In order to prove the equality, we have to prove that each manifold $L \in \mathcal{L}$ such that $H_{\mid L} \leqslant \alpha(H)$ contains $\tilde{\mathcal{A}}(H)$. This is almost the content of Theorem 5.1 in [14], except that we consider more irregular manifolds, which may not admit a genuine graph selector, and that we use a different definition for the Aubry set. Let us consider such a manifold $L$, and let us consider a point $\left(x_{0}, p_{0}\right)$ in $\tilde{\mathcal{A}}(H)$. Let $\Gamma \in \mathcal{G}$ be a Lipschitz exact Lagrangian graph contained in $\{H \leqslant \alpha(H)\}$. In other words, there exists a $C^{1,1}$ function $v: M \longrightarrow \mathbb{R}$ which solves the Hamilton-Jacobi inequality $H(x, d v(x)) \leqslant \alpha(H)$, and such that $\Gamma=\{(x, d v(x)), x \in M\}$. Since $\left(x_{0}, p_{0}\right) \in \tilde{\mathcal{A}}(H)$, we have $\left(x_{0}, p_{0}\right) \in \Gamma$, so that $p_{0}=d v\left(x_{0}\right)$.

Let us consider the function

$$
h(x)=\max _{p \in T_{x}^{*} M,(x, p) \in L} H(x,(p+d v(x)) / 2) .
$$

By the strict fiberwise convexity of $H$, we have $h(x) \leqslant \alpha(H)$, with equality if and only if $(x, d v(x)) \in L$ and $H(x, d v(x))=\alpha(H)$. It is therefore enough to prove that $h\left(x_{0}\right)=\alpha(H)$. We need the:

Lemma 2. The function $h$ is upper semi-continuous on $M$.

Proof. Let $x_{n}$ be a sequence of points of $M$ converging to $x \in M$, and let $p_{n} \in T_{x_{n}}^{*} M$ be such that $\left(x_{n}, p_{n}\right) \in L$ and such that $H\left(x_{n},\left[p_{n}+d v\left(x_{n}\right) / 2\right]\right)=h\left(x_{n}\right)$. We assume that the sequence $h\left(x_{n}\right)$ has a limit. By taking a subsequence, we can assume that $\left(x_{n}, p_{n}\right)$ has a limit $(x, p)$ in $L$, and then

$$
h(x) \geqslant H(x,[p+d v(x)] / 2)=\lim H\left(x_{n},\left[p_{n}+d v\left(x_{n}\right)\right] / 2\right)=\lim h\left(x_{n}\right) .
$$

We argue by contradiction and assume that $h\left(x_{0}\right)<\alpha(H)$. Then, there exists a smooth non-negative function $V(x)$ such that $h(x)+V(x) \leqslant \alpha(H)$ for all $x$, and such that $V\left(x_{0}\right)>0$. 
Let us then consider a generalized graph selector $u$ of $L$. For almost every $x$, the differential $d u(x)$ belongs to the convex hull of $L \cap T_{x}^{*} M$, so that

$$
H(x, d(u+v)(x) / 2) \leqslant h(x) .
$$

As a consequence, we have the inequality

$$
H(x, d(u+v)(x) / 2)+V(x) \leqslant \alpha(H)
$$

for almost every $x$. In other words, the functions $f=(u+v) / 2$ is a Lipschitz sub-solution of the Hamilton-Jacobi equation

$$
G(x, d f(x))=\alpha(H),
$$

where $G(x, p)=H(x, p)+V(x)$ is optical. Invoking once again the main result of [4], we infer that the same inequation has a $C^{1,1}$ subsolution $w$. This function satisfies

$$
H(x, d w(x))+V(x) \leqslant \alpha(H)
$$

for each $x$. The corresponding graph $\{(x, d w(x)), x \in M\} \in \mathcal{G}$ can't contain $\left(x_{0}, p_{0}\right)$ since $H\left(x_{0}, p_{0}\right)=\alpha(H)$ while $H\left(x_{0}, d w\left(x_{0}\right)\right)<\alpha(H)$. This is in contradiction with the assumption that $\left(x_{0}, p_{0}\right) \in \tilde{\mathcal{A}}(H)$.

\subsection{Exact diffeomorphisms}

It is tempting to define an Aubry set using the set

$$
\mathcal{L}=\{\varphi(\Gamma), \Gamma \in \mathcal{G}, \varphi \in \mathcal{E}\},
$$

where $\mathcal{E}$ is the set of all exact symplectic diffeomorphisms. The corresponding Aubry set is invariant under exact diffeomorphisms, and dynamically invariant. This theory would be interesting if Theorem 3 was true with this definition of $\mathcal{L}$, and we would have another proof of the full statement of Theorem 2. Unfortunately, we are not able to prove Theorem 3 with this definition of $\mathcal{L}$ at the moment. The problem is that we do not know whether a Lagrangian manifold which is the image of the zero section by an exact symplectic diffeomorphism admits any kind of generalized graph selector. As a consequence, we are not even able at the moment to prove that $\alpha_{\mathcal{L}}(H)=\alpha(H)$ for that generalized theory. Moreover, we do not know any class of Hamiltonians $H$ for which we can prove that $\tilde{\mathcal{A}}_{\mathcal{L}}(H)$ is not empty.

\section{References}

[1] M. C. Arnaud. Are pseudographs Lagrangian submanifolds? Nonlinearity, to appear.

[2] M. C. Arnaud. On a Theorem due to Birkhoff. GAFA, to appear.

[3] P. Bernard. Connecting orbits of time dependent lagrangian systems. Ann. Institut Fourier, 52(5):1533-1568, 2002.

[4] P. Bernard. Existence of $C^{1,1}$ critical sub-solutions of the Hamilton-Jacobi equation on compact manifolds. Annales scientifiques de l'Ecole normale supérieure, 40(3):445-452, 2007.

[5] P. Bernard. Symplectic aspects of Mather theory. Duke Mathematical Journal, 136(3):401-420, 2007.

[6] P. Bernard. The dynamics of pseudographs in convex Hamiltonian systems. J. A. M. S., 21(3):615$669,2008$. 
[7] M. Chaperon. Lois de conservation et géométrie symplectique. Comptes rendus de l'Académie des sciences. Série 1, Mathématique, 312(4):345-348, 1991.

[8] G. Contreras, R. Iturriaga, G.P. Paternain, and M. Paternain. Lagrangian graphs, minimizing measures and Mañé's critical values. Geometric and Functional Analysis, 8(5):788-809, 1998.

[9] A. Fathi. Weak KAM theorem in Lagrangian dynamics. ghost book.

[10] A. Fathi and A. Siconolfi. Existence of $C^{1}$ critical subsolutions of the Hamilton-Jacobi equation. Inventiones mathematicae, 155(2):363-388, 2004.

[11] A. Fathi and A. Siconolfi. PDE aspects of Aubry-Mather theory for quasiconvex Hamiltonians. Calculus of Variations and Partial Differential Equations, 22(2):185-228, 2005.

[12] R. Mané. Lagrangian flows: the dynamics of globally minimizing orbits. Bulletin of the Brazilian Mathematical Society, 28(2):141-153, 1997.

[13] J.N. Mather. Variational construction of connecting orbits. Annales de l'institut Fourier, 43(5):13491386, 1993.

[14] G.P. Paternain, L.V. Polterovich, and K.F. Siburg. Boundary rigidity for Lagrangian submanifolds, non-removable intersections, and Aubry-Mather theory. Moscow Mathematical Journal, 3(2):593$619,2003$.

[15] R. T. Rockafellar. Convex analysis, second edition. Princeton University Press, 1972.

[16] C. Viterbo. Symplectic topology as the geometry of generating functions. Mathematische Annalen, 292(1):685-710, 1992.

[17] C. Viterbo. Symplectic homogenization. Arxiv preprint arXiv:0801.0206, 2007. 